Musées, Patrimoine et Culture scientifiques et techniques

$165 \mid 2016$

mai-juin 2016

\title{
Quelle médiation pour les plages du Débarquement en Normandie?
}

\section{Stanislas Hommet et Myriam Chasserieau}

\section{(2) OpenEdition \\ Journals}

Édition électronique

URL : http://journals.openedition.org/ocim/1655

DOI : $10.4000 /$ ocim. 1655

ISSN : 2108-646X

Éditeur

OCIM

Édition imprimée

Date de publication : 1 mai 2016

Pagination : $19-23$

ISSN : 0994-1908

Référence électronique

Stanislas Hommet et Myriam Chasserieau, «Quelle médiation pour les plages du Débarquement en Normandie ? ", La Lettre de I'OCIM [En ligne], 165 | 2016, mis en ligne le 01 mai 2017, consulté le 19 avril 2019. URL : http://journals.openedition.org/ocim/1655 ; DOI : 10.4000/ocim.1655

Ce document a été généré automatiquement le 19 avril 2019

Tous droits réservés 


\section{Quelle médiation pour les plages du Débarquement en Normandie?}

Stanislas Hommet et Myriam Chasserieau

Véhicule militaire à Arromanches

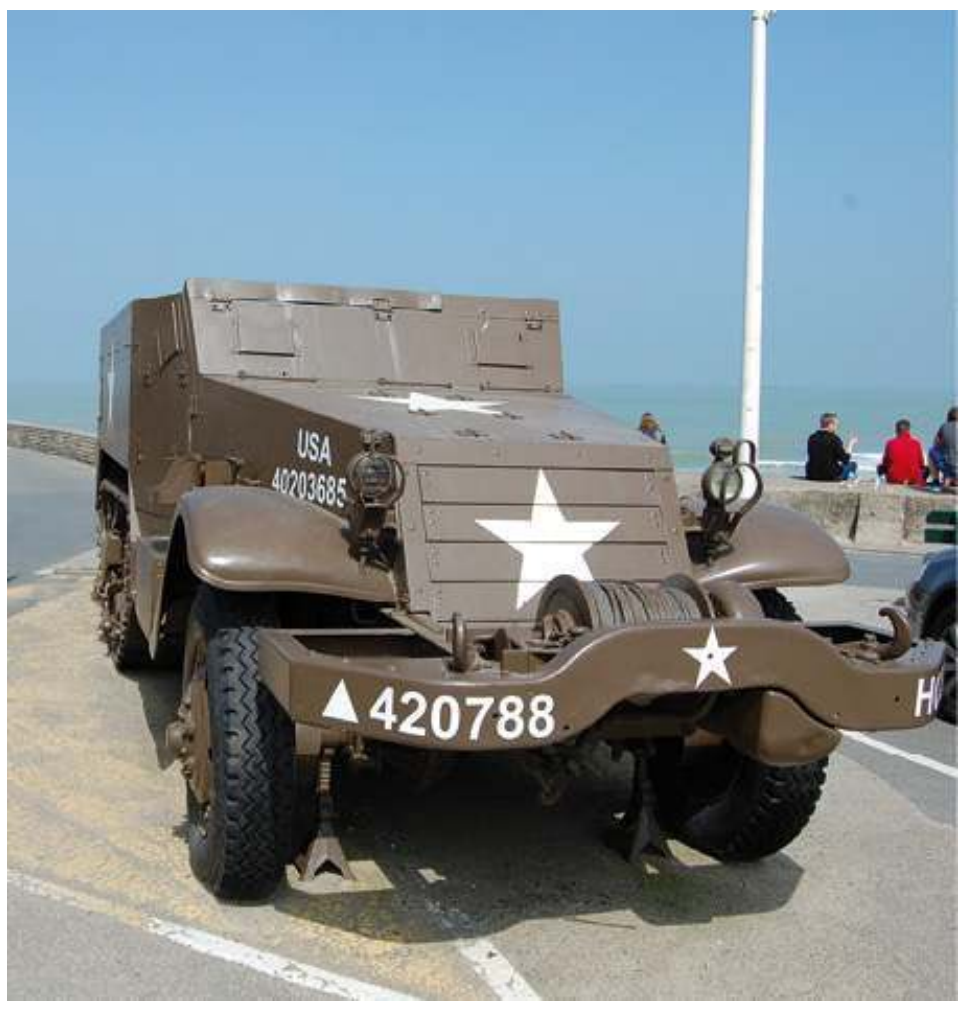

(c) S. Hommet 


\section{L'origine d'une étude}

1 La région Basse-Normandie s'est lancée depuis deux ans dans un projet de classement des plages du Débarquement au patrimoine mondial de l'Unesco. L'ambition est de faire du territoire la destination internationale d'excellence sur la Seconde Guerre mondiale, porteuse des valeurs universelles de liberté, de paix et de réconciliation. Ce projet constitue une opportunité de penser une évolution muséale, de construire un récit scientifique, et d'améliorer l'accompagnement du visiteur. À ce titre, la Région s'est tournée vers l'École supérieure du professorat et de l'éducation de l'université de Caen Normandie qui porte en son sein un parcours de formation de master 2 en médiation culturelle, pour mener une série d'études de réception en espace muséal.

2 Cet article présente les recommandations issues d'un diagnostic mené auprès de quinze sites historiques, musées, vestiges, centres d'interprétation et espaces mémoriels situés dans la zone à classer, parmi lesquels figurent des lieux qui, tels le cimetière américain de Colleville-sur-Mer et la Pointe du Hoc à Criqueville-en-Bessin, se placent parmi les plus visités de France. Ces différents sites constituent autant de témoignages de la présence militaire allemande, de l'assaut des troupes alliées sur les côtes normandes, et des affrontements de l'été 1944.

3 Ce travail a fait l'objet d'un stage de trois mois conduit, au printemps 2015, par une étudiante en master 2 médiation culturelle, Alexia Deneux-Chartrin, assorti d'un travail collectif. L'ESPE, à travers son parcours de formation en médiation culturelle, contribue à la réflexion des acteurs locaux pour aider à une mise en œuvre pédagogique d'une interprétation nécessaire. Ce diagnostic a été mené autour de quatre interrogations qui ont structuré l'observation et les entretiens ${ }^{1}$ - des interrogations ayant trait à l'appropriation de l'espace maritime du D-Day, à la relation entre l'expôt et le visiteur, à la construction d'un récit scientifiquement établi et harmonisé sur l'ensemble des sites, et à la mise en place d'une médiation digne de l'enjeu formidable que représente un tel classement. 


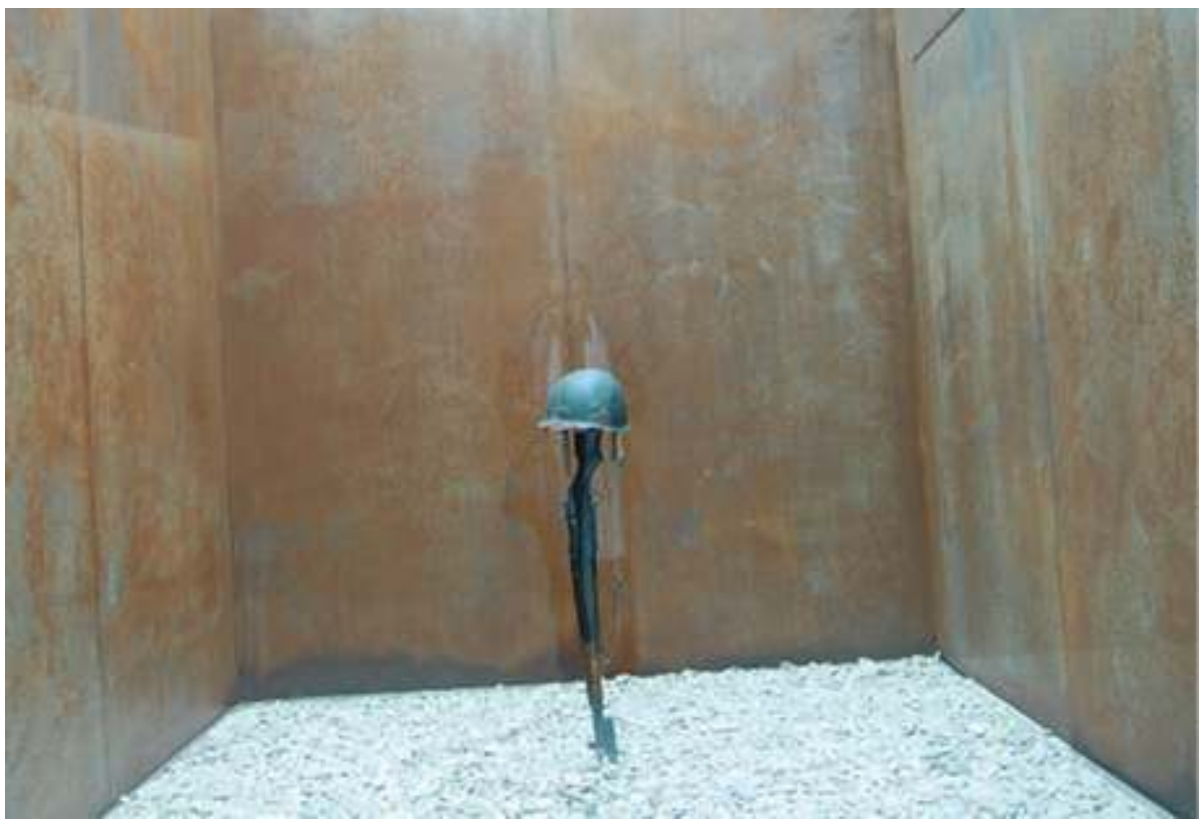

(c) S. Hommet

\section{Le visiteur destinataire et acteur de la scénographie : un objet muséal}

La mémoire du Débarquement et de la Bataille de Normandie s'inscrit dans des lieux emblématiques de l'assaut des troupes alliées: la région bas-normande compte près de quarante-cinq sites dédiés, partiellement ou entièrement, à ces événements. Les musées contribuent à offrir des clés de compréhension de cette période historique majeure. Si ces espaces muséographiques sont si nombreux, c'est qu'ils sont précisément issus d'une histoire et qu'ils sont eux-mêmes le reflet d'une mémoire de la Bataille de Normandie. Qu'ils soient en effet issus d'une collection privée rassemblée dès les années 1950, qu'ils aient vu le jour sous l'impulsion d'une volonté municipale, d'associations d'anciens combattants ou en hommage à un groupe de vétérans alliés, ou qu'ils soient, plus récemment, centres d'interprétation, les statuts des musées ne sont que le miroir de ces histoires locales, en étant le plus souvent le résultat d'une initiative locale : un musée des parachutistes qui fait suite à la volonté de Normands de garder les objets des soldats dès le lendemain de la bataille.

La diversité des musées est conséquente, ce qui explique, dès lors, la grande variété des approches des expôts, du récit scientifique présenté, et parfois, quand elle est identifiable, de la médiation.

Dans le cadre de l'observation, nous sommes partis d'un postulat prenant en compte le visiteur. Nous entendons par là que notre préoccupation porte sur la place du public dans les espaces muséographiques de la Bataille de Normandie. En effet, la région normande au travers de la Fabrique des patrimoines en place depuis le $1^{\text {er }}$ janvier 2015 et qui réunit trois associations, le CRéCET (Centre Régional de Culture Ethnologique et Technique), 1\&apos ;ARCIS (Agence Régionale de Conservation de l'Image et du Son) et Normandie 
Patrimoine (Centre régional de Conservation-Restauration des biens culturels de BasseNormandie) a pris en main les questions relatives à la conservation des objets, au respect des règles d'exposition.

7 Les sites liés au Débarquement et à la Bataille de Normandie accueillent chaque année plus de 2 millions de visiteurs. En 2014, année des commémorations du $70^{\mathrm{e}}$ anniversaire, ce sont près de 4 millions de visiteurs qui se sont rendus sur ces sites majeurs de l'histoire de la Seconde Guerre mondiale. Le profil des visiteurs tend cependant à évoluer. À mesure que les témoins disparaissent et que les liens familiaux s'évanouissent, les visiteurs ayant des intentions de pèlerinage, de souvenir ou de commémoration se font plus rares, laissant place à des visiteurs cherchant essentiellement à s'informer et à comprendre. Parce qu'à l'avenir, le visiteur ne sera plus aussi lié à cette histoire si douloureuse, il nous apparait essentiel de l'intégrer au centre de toute réflexion pour que la transaction réflexive attendue et espérée ait lieu. La nécessité d'adapter tant le récit que les outils de visite se fait d'autant plus forte que les sites sont amenés à accueillir une part croissante de visiteurs étrangers provenant non plus essentiellement du Royaume-Uni, des Pays-Bas, de la Belgique, de l'Allemagne, de l'Italie ou des États-Unis, mais également d'autres continents. Pétri d'expériences de lecture, de cinéma et de visite antérieures, le visiteur doit désormais prendre sa place : il devient non plus spectateur, mais acteur d'une visite qu'il mène, choisit selon ses expériences, ses connaissances et ses envies. Impliquer le visiteur constitue un préalable indispensable pour s'assurer de l'appropriation du message historique délivré.

\section{Des recommandations pour une véritable politique d'interprétation}

\section{Un espace maritime à valorise}

Les nouvelles techniques d'imagerie numérique et de relevé des fonds marins ont permis de révéler et de mieux appréhender la richesse du patrimoine sous-marin. Constitué d'épaves et de vestiges divers, ce patrimoine, imperceptible depuis les plages, demeure cependant largement méconnu. Le lieu du Débarquement des troupes alliées, répondant à des choix stratégiques précis, n'a pas été déterminé au hasard: l'espace maritime et les plages doivent, dès lors, constituer un élément fondamental du récit. Force est de constater que les plages ne disposent pas nécessairement de panneaux explicatifs et que, lorsqu'ils sont effectivement présents, les éléments d'information se révèlent trop précis, se limitent à l'espace visible, et ne sont que très rarement replacés dans le contexte plus large de l'opération Overlord. Une plage est représentative d'un aspect spécifique du Débarquement allié sur les côtes normandes, et il est dès lors indispensable que les dispositifs aménagés apportent des éléments permettant de nourrir l'interprétation des témoignages de l'événement replacé dans le contexte élargi de la Seconde Guerre mondiale. Par ailleurs, les dispositifs aménagés doivent conduire à la compréhension et à la satisfaction des besoins du visiteur néophyte. Entraîner le visiteur au-delà de ce qu'il perçoit : tel est l'enjeu qui doit être à la hauteur de ce précieux patrimoine subaquatique.

La difficulté de proposer une telle médiation doit amener les structures muséales à prendre en considération la dimension maritime dans leurs récits et leurs espaces scénographiques. Les structures muséales doivent être amenées à intégrer cette dimension dans leur propre exposition afin que les visiteurs trouvent des réponses à leurs 
interrogations. À cet égard, l'étude que nous avons menée au musée du Débarquement à Arromanches-les-Bains au printemps 2015 démontre que $80 \%$ des visiteurs se rendent sur la plage avant d'entrer dans le musée. Celui-ci propose notamment une série de maquettes présentant le port Mulberry B (le port artificiel) qui font écho aux vestiges qui marquent l'horizon, visibles de l'intérieur du musée. Elles contribuent à donner aux visiteurs des clés de compréhension du défi technique que fut la construction et l'exploitation du port artificiel. Dans les autres espaces muséographiques observés, la dimension maritime de l'opération Overlord n'est en revanche traitée que très partiellement. Il est fréquent de trouver aux abords et dans les halls d'entrée des musées, des objets-reliques liés aux opérations maritimes constituant autant de "traces ". Ces expôts sont cependant accompagnés d'indications trop souvent sommaires de leur nature, de leur origine, de leur provenance, et de leur rôle dans le contexte de la bataille. De même, la mise en place de péniches de débarquement à proximité du cinéma circulaire d'Arromanches 360, ouvert au printemps 2015, n'a pas eu pour suite une mise en valeur contextualisée permettant une réelle réflexion en matière de médiation adaptée à un public visiteur ignorant tout de leur fonction.

\section{Batterie de Longues-sur-Mer}

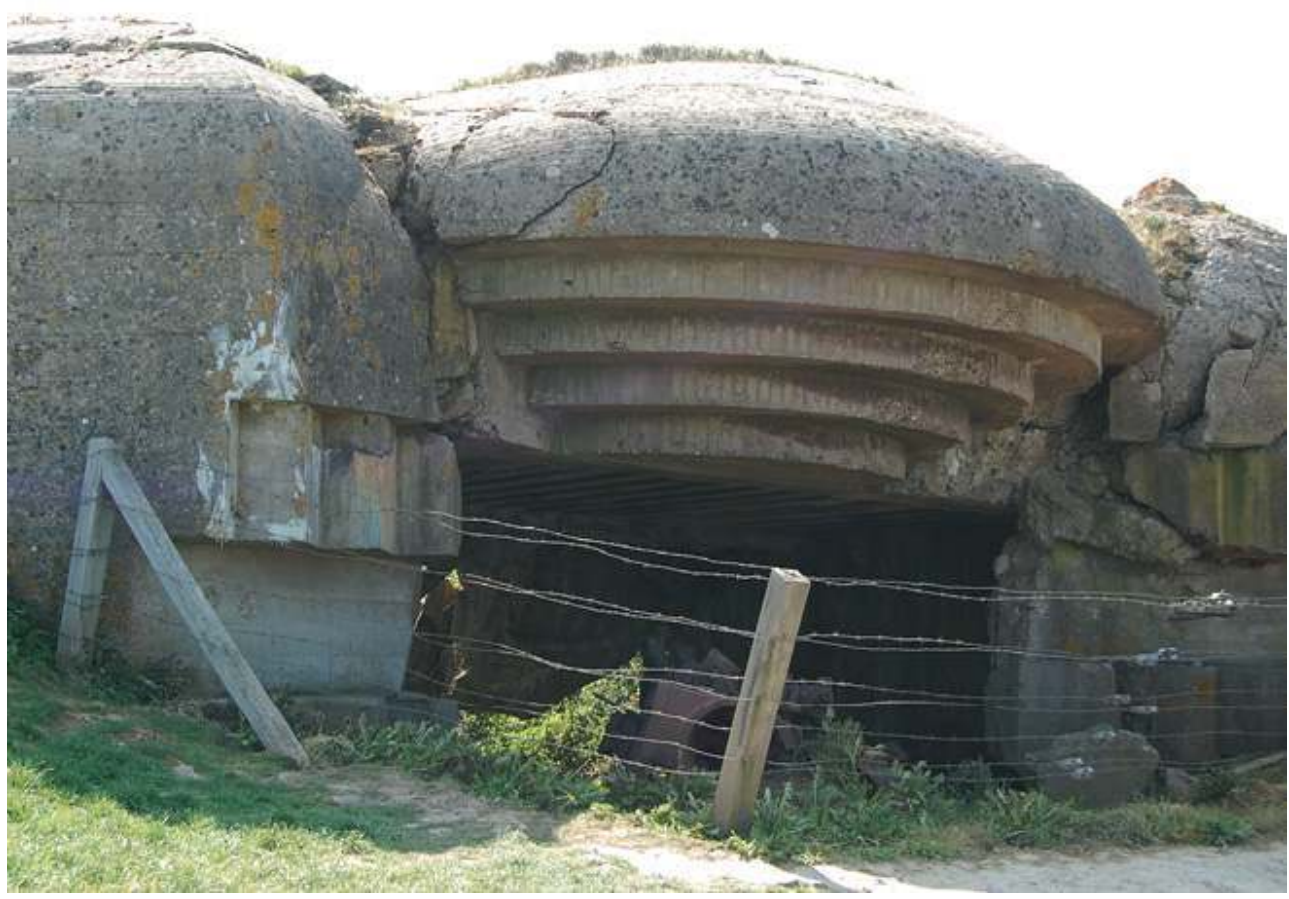

(c) A.-D. Chartrin

10 Mais les plages, ainsi que les paysages reliques durablement marqués par les combats, restent des lieux investis par des promeneurs qui n'auront la plupart du temps aucun contact avec un espace muséal, soit parce qu'ils limitent leur visite à l'extérieur, soit parce qu'aucun musée n'est situé à proximité. Ces expériences de visite nécessitent toute l'attention dans le cadre d'un classement Unesco et, dans ce contexte, le réseau de panneautage présent sur la zone à classer devient essentiel. Actuellement, les panneaux sont trop souvent détériorés et n'offrent que des explications parcellaires de l'opération Neptune et de la bataille qui s'ensuivit. La mise en place d'un site Internet dédié au site dans sa globalité devrait permettre également cette mise en contexte en fournissant au 
visiteur en amont, ou après, toutes les informations relatives aux plages du débarquement.

Afin de proposer une réelle réflexion, il convient dès lors de travailler la mise en contexte systématique des plages. Ainsi, deux axes seront à privilégier : la dimension maritime du débarquement et l'importance du patrimoine subaquatique invisible depuis la plage. Car si le visiteur se contente de rester en ces lieux, il n'aura en effet de lecture historique que celle qu'il aura préparée seul, en amont de sa visite. La promesse faite aux visiteurs tend à se limiter au seul accès à des sites uniques, témoins d'affrontements majeurs ayant conduit à la libération de l'Europe de la domination nazie.

Pour permettre au visiteur de préparer sa visite, il nous apparaît indispensable de construire un site Internet globalisant l'ensemble de la zone concernée par le classement Unesco. Ce site Internet constituerait l'espace de présentation de la Bataille de Normandie et offrirait un référencement des lieux à observer, des lectures conseillées, des fiches pédagogiques, ou encore des vidéos à télécharger. Il ne serait pas en situation de concurrence des sites Internet des structures muséales mais une interface entre les sites historiques et les musées d'une part, et les visiteurs d'autre part. L'individualisation de la visite que permettront les nouveaux outils de communication et d'information facilitera ainsi l'accompagnement du visiteur dans sa démarche réflexive.

Vitrine d'exposition à l'Overlord memorial Museum

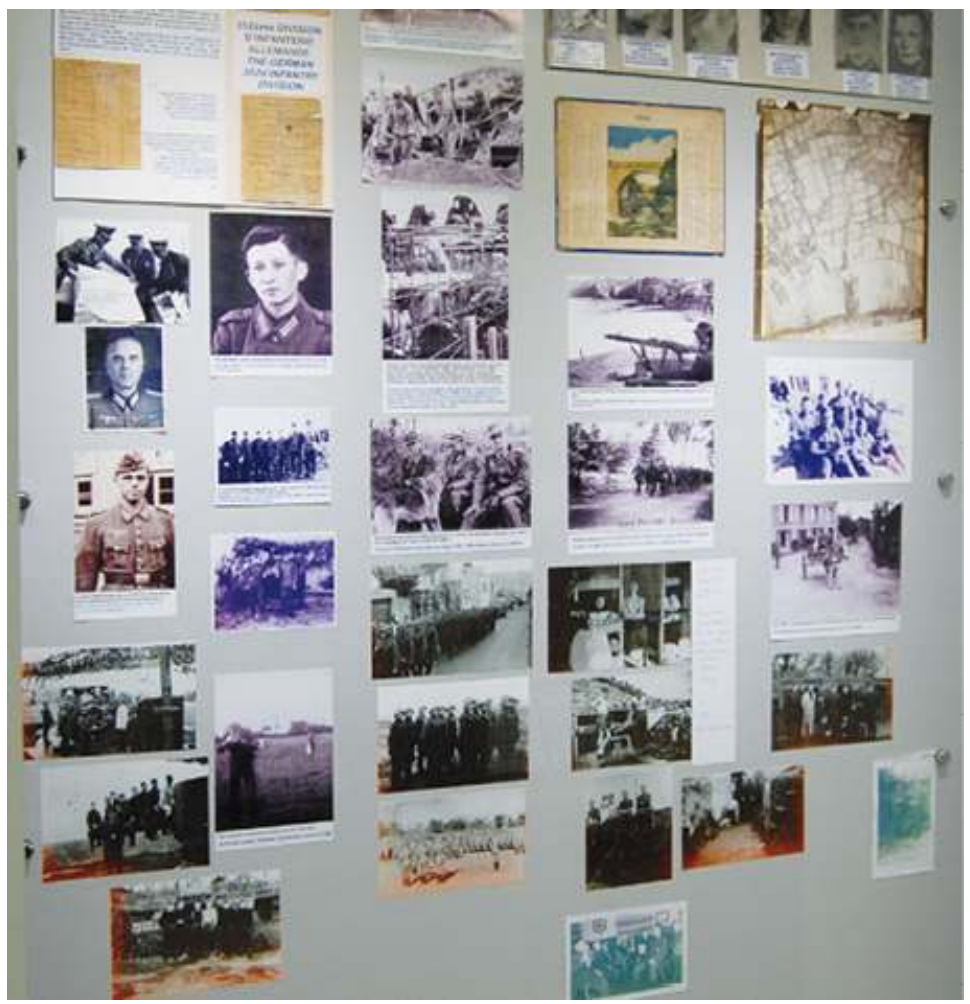

(c) A.-D. Chartrin

\section{L'expôt et le visiteur : une relation à établir}

Cette même préoccupation nous amène à penser qu'il convient de saisir l'opportunité du classement pour mener une évolution muséale nécessaire en pensant la relation entre 
l'objet exposé et le visiteur. L'histoire des collections, et ce même si elles demeurent incomplètes, mérite d'être valorisée dans l'ensemble des musées de la zone concernée par le classement. Et à juste titre : derrière chaque collection, c'est une histoire particulière qui mérite d'être connue et qui contribue à expliquer pourquoi ce casque percé se trouve précisément à cet endroit et pourquoi cette robe conçue à partir d'une toile de parachute est exposée dans cette vitrine ${ }^{2}$. Cet aspect, qui s'inscrit dans la mémoire de la Bataille de Normandie, présente un intérêt réel pour le visiteur qui cherche aussi à comprendre pourquoi c'est ici-même, dans cette région, que ces événements se sont très précisément déroulés. À cet égard, la présence de nombreux monuments commémoratifs rappelle les liens qui unissent les anciens pays belligérants au territoire normand, renforçant ainsi son caractère unique.

ex la diversité des objets exposés dans l'ensemble des musées de la zone à classer est manifeste. Cependant, ils demeurent trop souvent perdus entre une scénographie qui se voudrait immersive et une scénographie d'idées, quand la mise en récit ne se révèle pas être tout simplement absente. Pourtant, leur place mérite d'être enfin pensée : combien de vitrines rassemblant de nombreux expôts dépourvus de cartels ne font l'objet que d'un simple regard périphérique du visiteur ? Le classement des plages du Débarquement au patrimoine mondial de l'Unesco, mérite avant toute chose un travail en profondeur sur les objets présents dans les musées - nature, provenance, état de conservation, conditions d'exposition, médiation vers l'expôt. Enfin, le paysage physicomémoriel aux abords des musées, mérite d'être travaillé : il n'est pas rare de trouver des objets-reliques parfaitement restaurés et entretenus, disposés aux abords d'un musée, sur un parking ou sur la route dans le seul but d'évoquer les événements et de susciter la curiosité du visiteur.

Ces objets-reliques sont autant de preuves collectées pour attester, de manière tangible, des opérations militaires qui se sont précisément déroulées sur le territoire. Les sites et les musées sont habités par un souci d'authenticité : authentiques, les objets le sont parce qu'ils ont été retrouvés sur les plages de Normandie, parce qu'ils appartenaient à un soldat identifié ou tout simplement parce qu'ils étaient contemporains des événements. Le visiteur participe à une rencontre produite par des objets dans le but de vivre une expérience unique. Si le caractère exceptionnel de ces objets est indéniable, ils ne suffisent toutefois pas à favoriser la compréhension historique puisqu'ils sont peu insérés dans un récit explicatif. En effet, chaque objet possède en lui plusieurs dimensions, notamment ce qu'il dit de façon générale d'événements historiques, mais aussi son histoire propre. Leur potentiel est sous-estimé, dans la mesure où ils constituent trop souvent des éléments d'une collection qui vaut par son ampleur ou sa spécificité. Les expôts, non plus seulement objets signifiants, doivent devenir des leviers pour interroger le visiteur et susciter une réflexion sur un récit historique.

\section{Un récit scientifique à construire}

Les espaces muséographiques invitant à une telle réflexion sont encore trop rares. Nombre d'entre eux trouvent leur origine dans une collection à mettre en valeur et privilégient une approche chrono-thématique, le sens de l'exposition émanant ainsi essentiellement des objets exposés. Dans le cas d'une muséographie d'objets, le visiteur utilise sa propre expérience de visite et mobilise ses connaissances pour situer, comparer et donner du sens à ce qu'il observe quand le sens n'est pas intrinsèquement donné. Des 
objets liés à l'armée allemande et aux forces armées alliées se confondent parfois dans le même espace d'exposition, ce qui pose notamment le problème de l'ordonnancement des expôts.

Le récit doit laisser une large place à une prise de distance réflexive pour amener le visiteur à s'interroger sur les valeurs de paix, de réconciliation, de sacrifice et de liberté qui sont au cœur du projet de classement des plages du Débarquement.

Ce constat et ces recommandations sont logiquement liés au fait que les commissions scientifiques sont inexistantes dans la grande majorité des musées. Si certains font appel à des historiens locaux, parfois des historiens militaires étrangers, rares sont ceux qui ont constitué un comité d'experts scientifiques pour construire leur récit et penser les évolutions de l'exposition. Notons, qu'à ce jour, la première étape pour les musées souhaitant s'agrandir, repose sur la mise en place d'un comité scientifique. Le musée du Débarquement à Arromanches-les-Bains, qui travaille à une refonte de ses espaces, a d'abord mis en place un conseil scientifique avant de faire appel à un programmiste. De même, la commune de Ouistreham, qui se pose la question d'un centre d'interprétation sur son territoire, a constitué un tel conseil.

\section{Une médiation à renforcer}

Enfin, la médiation se révèle inexistante dans la zone concernée par le classement, et les outils à destination d'un public étranger et d'un public scolaire restent à concevoir. Pourtant, la médiation est au cœur de tout le processus nécessaire d'élaboration du récit historique. Il est indispensable que, dès la conception des contenus, les médiateurs professionnels interviennent pour identifier les clés du récit. Dans un second temps, des outils numériques et de scénographie sont à utiliser pour faciliter la transaction réflexive - cette rencontre entre l'objet social qu'est le musée et le public. Le visiteur, considéré comme un objet muséal, doit concentrer toute l'attention du musée - ses déplacements tout autant que sa réception de l'exposition. Les outils de médiation doivent permettre au visiteur considéré comme objet muséal de se questionner, de chercher à comprendre et de s'exprimer.

Les musées observés sont tous demandeurs d'un travail de fonds sur la notion même d'interprétation et souhaitent être accompagnés dans le cadre du classement pour progresser et permettre au public visiteur de vivre une réelle expérience de visite au service de son questionnement. Ainsi, dans le cadre de son contrat de destination "tourisme de mémoire", la région Normandie incite les structures muséales à s'engager dans un travail réflexif quant à l'offre de médiation disponible pour tous les publics.

21 L'École supérieure du professorat et de l'éducation de l'académie de Caen qui porte en son sein un parcours de formation de master en médiation culturelle, contribue à cette réflexion en menant des audits sur les outils de médiation en place. En effet, l'exigence du classement doit permettre à la région Normande de repenser ses outils de médiation, mais également de concevoir que la médiation constitue un corps de métier propre aux compétences ciblées et qu'il est plus qu'utile de recruter des personnes formées à la médiation. 
Panneau d'interprétation de la Batterie de Longues-sur-Mer

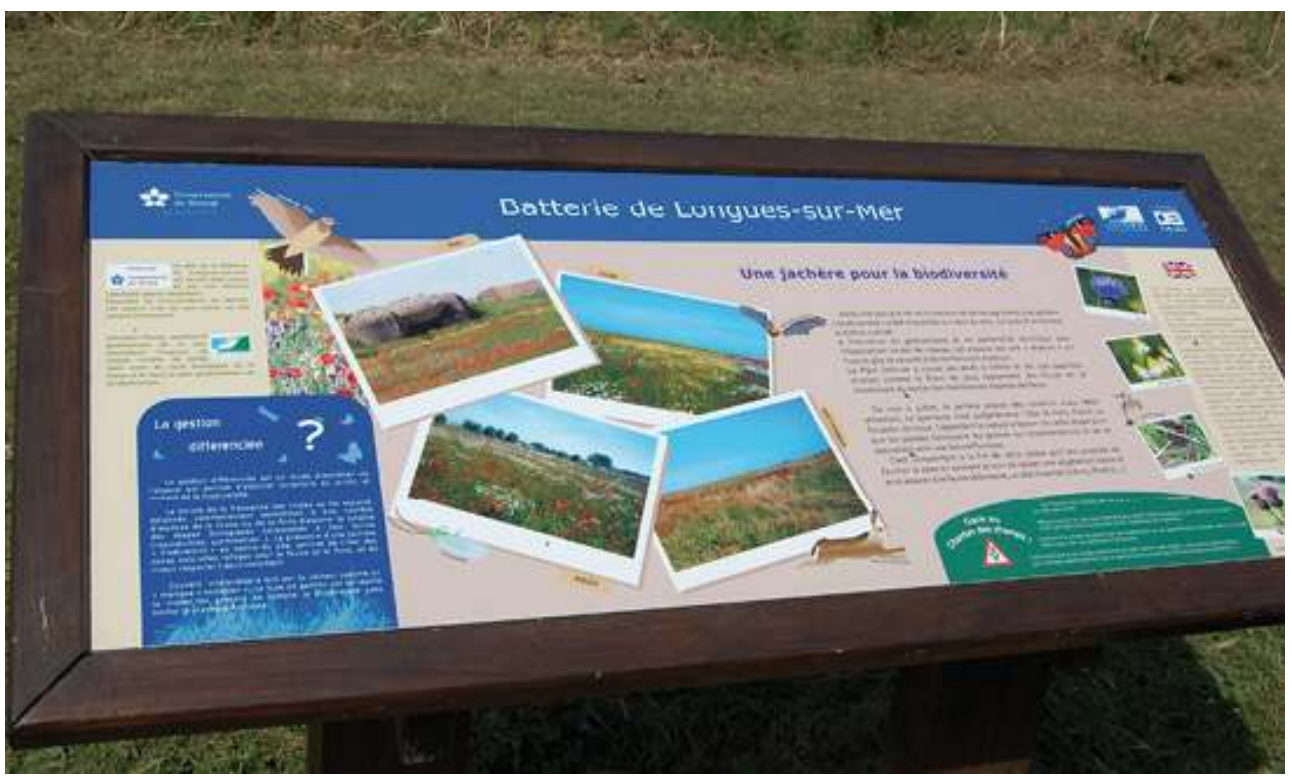

(c) A.-D. Chartrin

Doter tous les musées de la zone à classer d'outils de médiation dignes d'intérêt et prenant en compte les publics en répondant aux exigences que nous exposons ici, mais aussi élever le niveau de qualification et de compétences des personnels engagés dans la médiation, tels sont les ambitions nécessaires pour imaginer offrir au public une interprétation à la hauteur des enjeux des combats de 1944.

\section{BIBLIOGRAPHIE}

Chaumier, S. La médiation culturelle. Paris : Armand Colin, 2013, 284 p.

Daignault, L. L'évaluation muséale. Québec : PUQ, 2011, 348 p.

Daignault, L. Dépasser ou réinventer l'évaluation, in Daignault, L. et Schiele, B. (dir.) Les Musées et leurs publics, savoirs et enjeux. Québec : PUQ, 2014, pp. 71-83.

Hommet, S. Rapport d'étude sur l'interprétation sur les sites à classer au patrimoine mondial de l'Unesco des plages du Débarquement. www.unicaen.fr/espe

\section{NOTES}

1. Le détail de la méthode d'enquête est exposé dans le rapport d'étude L'interprétation sur les sites à classer au patrimoine mondial de l'Unesco, consultable sur www.unicaen.fr/espe

2. Plusieurs musées de la région exposent en vitrine de telles robes de mariées fabriquées en toile de parachute. 


\section{RÉSUMÉS}

À la suite de la demande de classement des plages du Débarquement en Normandie au patrimoine mondial de l'Unesco, un audit mené par l'École supérieure du professorat et de l'éducation de Caen a permis d'effectuer un diagnostic auprès des différents établissements culturels et patrimoniaux concernés et de proposer un certain nombre de recommandations destinées à la mise en place d'une véritable politique d'interprétation de ces lieux.

INDEX

Mots-clés : médiation

\section{AUTEURS}

\section{STANISLAS HOMMET}

directeur de l'ESPE de l'académie de Caen, vice-président de l'université de Caen Normandie, responsable du parcours de Master 2 médiation culturelle, chercheur au laboratoire ThéodileCirel de l'université de Lille 3

stanislas.hommet@univcaen.fr

\section{MYRIAM CHASSERIEAU}

docteure en études anglophones (Aix-Marseille Université), chargée de médiation dédiée à la valorisation de la recherche, université de Caen Normandie myriam.chasserieau@univcaen.fr 